\title{
EXPERIENCIAS E IMPACTO SOCIAL EN LA APLICACIÓN DE INGENIERÍA DE REQUISITOS EN EL DESARROLLO DE UNA PLATAFORMA INTELIGENTE
}

\author{
Gladys Maquera $^{1 \mathrm{a}}$, Jesús Mariaca ${ }^{2}$, Óscar Mendoza ${ }^{3}$ y Nelly Condori-Fernandez ${ }^{4}$ \\ Escuela Profesional de Ingeniería de Sistemas, Universidad Esan, Perú ${ }^{1}$,Universidad \\ peruana Unión ${ }^{2}$, \\ Grupo Innop Perú E.I.R.L., , Juliaca, Perú ${ }^{3}$ \\ Universidade da Coruña ${ }^{4}$ \\ Orcid ID: https://orcid.org/0000-0001-7308-4952 ${ }^{1}$ \\ Orcid ID: https://orcid.org/0000-0003-4798-250X ${ }^{2}$ \\ Orcid ID: https://orcid.org/0000-0003-3861-74123 \\ Orcid ID: https://orcid.org/0000-0002-1044-3871 ${ }^{4}$
}

Recibido: 15 de octubre de 2018

Aceptado: 07 de enero de 2019

\begin{abstract}
Resumen
El turismo rural comunitario (TRC) es una actividad que contribuye, desde las zonas rurales, al desarrollo económico-social y sostenible de un país. En este trabajo de investigación se tuvo como objetivo desarrollar una Plataforma Inteligente (PI) que satisfaga las necesidades de los distintos actores del TRC. Se presenta la experiencia centrada en Ingeniería de requerimientos, pasando por distintas iteraciones, donde se realizó el análisis FODA, uso de la programación extrema, cumplimiento de la ISO 25010, entre otros. La identificación precisa de los requerimientos fue determinante para que la implementación y usabilidad de la plataforma tenga un impacto social en los actores directos.

Palabras clave: Informe de experiencia, turismo rural comunitario, ingeniería de requisitos, plataforma inteligente.

\section{EXPERIENCES AND SOCIAL IMPACT IN THE APPLICATION OF REQUIREMENTS ENGINEERING IN THE DEVELOPMENT OF AN INTELLIGENT PLATFORM}

\begin{abstract}
Rural community tourism (RCT) is an activity that contributes, from rural areas, to the socioeconomic and sustainable development of a country. The purpose of this research study was to develop an Intelligent Platform (IP) that meets the needs of the different stakeholders of the RCT. It presents the experience focused on requirements engineering, going through different iterations, where the SWOT analysis was performed, along with the use of extreme
\end{abstract}


programming, compliance with ISO 25010, among others. The precise identification of the requirements was crucial for the implementation and usability of the platform to have a social impact on the direct actors.

Keywords: Experience report, rural community tourism, Requirements engineering, intelligent platform.

\section{Introducción}

El Turismo Rural Comunitario (TRC) se viene consolidando como una alternativa social justa, ambientalmente responsable. Sin embargo, en este sector se encuentra diferentes dificultades; como por ejemplo, la participación, respuesta de los actores y promoción de productos turísticos suele ser de tipo tradicional lo que genera informalidad en la gestión de la información entre los actores del TRC, la visibilidad en la web de los recursos/atractivos turísticos es incipiente y de los que existen no tienen ventajas competitivas, no existe la información básica de recursos y productos turísticos para el turista y mucho menos que se le pueda sugerir una ruta que satisfaga sus necesidades. Por otro lado, los buscadores de internet ofrecen: productos de viaje tradicionales, como pasajes aéreos, alojamiento, restaurantes y movilidad para alquilar a lo que se le llama el "eTurismo" (turismo electrónico), que según Buhalis (2003) es la digitalización de todos los procesos y cadenas de valor en las industrias de turismo, viajes, hostelería y restauración que permiten a las organizaciones maximizar su eficiencia y eficacia.

Sin embargo, el uso de internet plantea nuevos desafíos para la industria del turismo; es decir, para los distintos tipos de consumidores: Generación Silenciosa, los BabyBoomers, la Generación X y la Generación Y (Gomez, 2011; Maquera, Mariaca y Mendoza, 2016). La generación Y, son los más activos y mucho más involucrados en la planificación de viajes por internet, utilizan una variedad de información, dispositivos de comunicación, hacen reservas en línea con más frecuencia, muestran mayor uso de viajes online, consideran destinos a visitar; buscan activamente qué actividades pueden ser realizadas, lugares para ir de compras y donde comer, buscan una amplia gama de experiencias turísticas incluyendo festivales, eventos y espectáculos deportivos; ellos son muy abiertos y receptivos a la publicidad online, Maquera et al (2016).

Según Kim, Kandampully y Bilgihan (2018), el 56\% toman como base los comentarios y experiencias de familiares y amigos, lo que fortalece la denominada "eWOM", es decir, bocaa-boca electrónico. En la actualidad, en el Perú, el Turismo Rural Comunitario (TRC) se viene consolidando como una alternativa social justa, ambientalmente responsable. En este trabajo de investigación se presenta una experiencia al construir una Plataforma Inteligente (PI) que 
integra a los distintos actores del TRC; así como se muestra la experiencia de usabilidad de la plataforma en los emprendedores, actores sociales, que en algunos casos nunca tuvieron contacto con una computadora.

\section{Principales problemas y propuestas de soluciones}

En esta sección se da énfasis a la visión general del TRC así como de la plataforma, los procesos identificados en la ingeniería de requisitos, aplicación del análisis FODA, Qiufen (2012) para la identificación de los requisitos de la plataforma inteligente, acciones de mejoría y el impacto que se tuvo al identificar correctamente los requisitos y por ende la implementación de la plataforma.

\section{Turismo rural comunitario}

El turismo rural comunitario es toda actividad turística que se desarrolla en el medio rural, de manera planificada y sostenible, basada en la participación de las poblaciones locales organizadas para beneficio de la comunidad, siendo la cultura rural un componente clave del producto. Su objetivo es contribuir, desde las zonas rurales, al desarrollo de un Turismo sostenible como herramienta de desarrollo económico-social del Perú, MINCETUR (2019).

En el Turismo Rural Sostenible, la participación de la comunidad local es una componente importante de la actividad turística, se encuentra regulada por la Ley $\mathrm{N}^{\mathrm{o}} 29408$, Ley General de Turismo, y su reglamento aprobado por D.S. No 03-2010. Tiene por objeto promover, incentivar y regular el desarrollo sostenible de la actividad turística. En relación al uso de las TICs a nivel de América Latina existen plataformas relacionadas, entre ellas destacamos la de TripAdvisor ("Consejero de Viaje") inicialmente sus usuarios compartían sus experiencias en relación a hoteles, restaurantes y recomendaciones para sus viajes, hoy en día permite comprar y hacer la reserva de hoteles y pasajes aéreos. La plataforma de la Asociación Italiana de turismo responsable (http://www.aitr.org), el consorcioCTM AltroMercato (http://www.altromercato.it/) son protagonistas en la promoción y realización de iniciativas de la economía solidaria en Italia. En el Perú tenemos el portal http://www.turismoruralcomunitario.gob.pe/ donde se da información básica de los lugares donde se ha implementado el TRC. Sin embargo; ninguna de ellas es una Plataforma Inteligente en que el turista, además de tener información básica, pueda obtener una ruta inteligente (conforme a sus requerimientos), oferte productos turísticos valorando la gastronomía y/o 
artesanía o que integre alguna cadena productiva que pueda generar competitividad a la región y muestre recursos y atractivos turísticos, Maquera et al (2016).

Para el desarrollo de la PI se realizó un análisis de los requerimientos básicos, las funcionalidades deseadas, así como otras áreas, tales como la arquitectura, base de datos e interfaz de los distintos usuarios para ser validados por los distintos actores del TRC. Posteriormente se realizó el análisis FODA para conocer el contexto actual del TRC; y se identificó los procesos que suceden en el TRC; así como se identificaron los módulos de la Plataforma y principalmente las funcionalidades deseadas de la plataforma para el uso de los actores directos.

\section{Análisis FODA}

Una de las herramientas utilizadas en planificación estratégica y que ayuda a la toma de decisiones para trazar la trayectoria futura de las organizaciones, es el análisis FODA (Fortalezas, Oportunidades, Debilidades y Amenazas), Qiufen (2012). Se dio énfasis a las fortalezas y a las oportunidades, esto con el objetivo de presentar un modelo de gobierno electrónico para el TRC, considerando las distintas necesidades de los actores directos, normativas y tendencias del turismo.

Entre las principales fortalezas que se consideró para implementar la plataforma tenemos: existencia de asociaciones de TRC, constituidas con experiencia, existencia de recursos turísticos potenciales que aún no fueron puestos en valor, pero que sí reciben visitas esporádicas, así como la recuperación de conocimientos ancestrales (artesanía, gastronomía, plantas medicinales) y respeto al medioambiente. Entre las oportunidades que se identificaron están: unir la productividad con el TRC, disminución de la pobreza, mitigación del cambio climático y generación de empleo con los emprendimientos que se implementen.

Con el conocimiento de las fortalezas y de las oportunidades en el TRC es pues que se inicia el trabajo de identificar los requisitos funcionales de la plataforma, de tal manera que satisfaga las necesidades de los distintos actores. El conocimiento de las debilidades y de las amenazas existentes en el TRC, nos ayudó para complementar la implementación de la plataforma y posterior uso de los actores; es decir, se trabajó intensamente en la gestión del TRC e implementación del TRC para tener el respectivo impacto social y direccionando para que los actores, posteriormente utilicen la plataforma. 


\section{Business process modeling}

Para identificar los procesos y modelarlos se utilizó Business Process Management que es un conjunto de métodos, herramientas y tecnologías utilizados para diseñar, representar, analizar y controlar procesos de negocio operacionales, Osterwalder y Pigneur (2009). Entre los pasos seguidos tenemos:

- Identificar los procesos: En esta etapa se hizo un análisis de los procesos que existen y se ejecutan en los diferentes actores visitados, se utilizó diversas técnicas como visitas en las áreas, listas tareas de las áreas o personas, entrevistas; así como los deseos que las personas tenían en relación a su función.

- Identificar los propietarios de los procesos: En esta etapa se identificó a las personas involucradas con los procesos (dueños de los procesos), principalmente aquellos que se encargaban de realizar dichos procesos.

- Mantener la relación entre los procesos: Se asumió que los procesos de los negocios no son aislados, sino que mantienen un cierto nivel de relación entre ellos, por lo tanto, se dio énfasis al momento de representar los procesos.

- Documentar: Todas las etapas del modelamiento de procesos se documentaron, registrando las evidencias para que los procesos identificados, posteriormente sean revisados, analizados y validados. Es necesario resaltar que la documentación no necesariamente sólo fue documentación física, sino que digital.

Entre los procesos identificados, inicialmente, se tiene: proceso de reserva de paquetes turísticos - Turista Independiente, proceso para que un emprendimiento potencial se convierta en un Real, proceso de creación de proyectos sociales (turismo solidario-voluntario), proceso de mantenimiento y mejora de los emprendimientos, proceso para la creación de servicios, proceso de censo de turistas.

\section{Obtención de requisitos, validación y priorización.}

Con la finalidad de identificar los requisitos funcionales de la plataforma en TRC se realizaron visitas a diferentes experiencias a nivel nacional e internacional, así como instituciones, proyectos públicos y privados que vienen trabajando con este tipo de turismo; en las cuales, por medio de reuniones, entrevistas (previa comunicación por teléfono) y otros se logró extraer información primordial para obtener el know how que se necesitaba para la 
realización de la plataforma. También se recaudó información relevante de artículos científicos relacionados, informes, sitios web oficiales, y documentos oficiales en general.

Una de las metodologías ágiles para el desarrollo es Extreme Programming, la cual es defendida por Beck y Andres (2005) entre otros; esta metodología se compone de un conjunto de valores y prácticas importantes que forman un método para el desarrollo de software. Dentro de estas prácticas se encuentra la creación de Pruebas de aceptación, las cuales representan algún resultado esperado del sistema, esto garantiza que se cumplan los requisitos, logrando que el sistema sea aceptable. Consideramos 5 etapas para la identificación de los requisitos; i) priorización de requisitos, luego de la identificación de los requisitos, se priorizó la lista de requisitos de la plataforma digital, esto se realizó para tener una secuencia de requisitos a implementar y tener un orden establecido al momento de desarrollar la plataforma; ii) planificación de la primera iteración, se seleccionó tres requisitos para la primera iteración del desarrollo de la plataforma; se realizó el listado de las tareas que deben realizarse durante un tiempo establecido que duró la primera iteración. Una vez culminado ese tiempo, se entregó los 3 requisitos implementados y aptos para poder realizar pruebas beta de la plataforma; iii) análisis de los requisitos de la primera iteración, teniendo la lista de tareas, se realizó el diagrama de clases de los requisitos seleccionados, para lo cual se identificó las clases involucradas en la primera iteración y se analizó las relaciones entre estas. iv) preparación del repositorio base del proyecto, se elaboró la estructura de la plataforma de tal manera que sea escalable y fácil de dar mantenimiento, utilizando tecnologías de desarrollo ágil. Toda la estructura inicial se transfirió a un repositorio privado para que pueda ser accesible por los miembros del equipo de desarrollo y v) desarrollo del requisito "Registro de Recurso Turístico", se inició con el desarrollo del "backend" de este requisito. Posteriormente se realizó el desarrollo del "frontend"; el cual podrá ser visualizado desde cualquier navegador.

\subsection{Acciones de mejoría}

Luego de identificar los requisitos funcionales de la plataforma, en su primera iteración; así como identificar los procesos, nos enfocamos en el sitio web. La mayoría de usuarios rechazan un sitio web por su apariencia, esto no se trata sólo de tener un sitio web "bonito" y nada más. Realmente, qué tan bien luce un sitio web es subjetivo, pero lo que no es relativo es el orden, la usabilidad y la experiencia de usuario. Entonces en una segunda iteración de la identificación de los requisitos para la plataforma se dio énfasis a la usabilidad y experiencia de usuario. 
La definición que la norma internacional ISO 25010 hace sobre "usabilidad" expone que es "la capacidad del producto software para ser entendido, aprendido, usado y resultar atractivo para el usuario, cuando se usa bajo determinadas condiciones". Es decir, es la facilidad que tienen los usuarios de navegar y de acceder a los contenidos de una web. Y la experiencia de usuario (UX) según la norma ISO 9241-210, es “el resultado de las percepciones y respuestas de una persona por el uso y uso anticipado de un producto, sistema o servicio".

Para mejorar la usabilidad y experiencia de usuario del sitio web de la plataforma, se tuvo que realizar un nuevo diseño del sitio web, el cual fue desarrollado por un equipo especializado con experiencia en estos temas, en esta oportunidad se identificaron nuevos requisitos funcionales para la usabilidad de la plataforma por parte de los actores denominados "emprendedores rurales de centros productivos", quienes son los que ofrecen sus productos y servicios a los demás actores. Se obtuvo como producto final archivos desarrollados en el programa Photoshop, los cuales se tuvieron que implementar en la web de la plataforma. Terminada la implementación se desarrolló e implementó la internacionalización en las URLs del sitio web. Este nuevo diseño desarrollado, brindaría una mejor usabilidad y experiencia de usuario a los emprendedores; así como al visitante web.

Posteriormente, en una última iteración, se tuvo una capacitación de la usabilidad de la plataforma a quienes serían los orientadores turísticos locales, formados por miembros de las comunidades indígenas, quienes no conocían la existencia de plataformas semejantes y nunca tuvieron acceso al uso de computadoras, pero sí de celulares con redes sociales. Inicialmente, se les enseñó el uso de otras plataformas, para que, con ese conocimiento adquirido, sean ellos los que vean la diferencia con nuestra plataforma. Se identificaron nuevos requerimientos de usabilidad para los orientadores turísticos locales, entre ellos está, dar mayor información de los recursos/atractivos turísticos para que ellos puedan aprender la información turística y de este modo dar mejor orientación al turista.

\section{Impacto de los requerimientos}

Con los requisitos funcionales implementados en la plataforma se inicia la promoción y difusión de la plataforma, principalmente entre los actores directos (emprendedores, orientadores turísticos locales y turistas), el siguiente paso a seguir es promocionar y difundir la plataforma en las agencias de viajes y turistas de ecoturismo a nivel mundial. También es necesario mencionar que el Ministerio de Comercio Exterior y Turismo y PROMPERU tienen conocimiento de la plataforma. Por otro lado, mientras se desarrollaba la plataforma el equipo 
de investigación, conoció a otros actores en promocionar el TRC, ninguno de esos proyectos, así como instituciones habían considerado el uso de las TICs y mucho menos el de implementar una plataforma específica para el TRC; así como el de capacitar en TICs a emprendedores rurales. Es importante destacar, que este trabajo de investigación fue integral y los otros proyectos e instituciones (proyecto SAYWA de la Unión Europea, MINCETUR, DIRCETUR y Municipalidades locales) comenzaron a seguir este modelo que fue difundido por redes sociales.

La plataforma denominada CAMINOS DEL INKARRI, aún sigue siendo única a nivel de América del Sur, por trabajar con Inteligencia Artificial, Investigación de Operaciones y Ciencia de Datos.

\section{Lecciones aprendidas e impacto social}

La identificación precisa de los requerimientos funcionales principales es fundamental para el cumplimiento de las metas y objetivos trazados; es necesario no sólo concentrarse en lo actores directos del uso de la plataforma, sino también en lo de los indirectos. Inicialmente no tuvimos el apoyo de todos los actores y tampoco la confianza de los que trabajaban con nosotros, para conseguir lo que deseábamos tuvimos que trabajar en tareas complementarias; es decir, en áreas sociales, ayudarles a implementar el TRC. Utilizar buenas prácticas de gestión de requerimientos, reduce el tiempo para la obtención de la información necesaria y suficiente. La retroalimentación de los requisitos es fundamental para tener la especificación adecuada; así como relacionarse directamente con los actores comunitarios y ayudarles, en otras áreas y principalmente hacerles sentir que ellos son "dueños" de la plataforma (por considerar sus sugerencias) asegura el éxito y por ende la fidelización para el uso de la plataforma.

\section{Conclusiones y trabajos futuros}

Se ha identificado los requerimientos funcionales necesarios y suficientes de una plataforma inteligente para el turismo rural comunitario. Esta identificación permitió la implementación y usabilidad inmediata de la plataforma por parte de los actores directos y durante el desarrollo del trabajo de investigación; así como ha generado el interés por parte de instituciones público y privadas, por ser una plataforma que considera la Inteligencia Artificial, Investigación de Operaciones y Ciencia de datos. 
Entre los trabajos a futuro que se tiene, es el de implementar otros módulos, tales como el turismo voluntario, turismo científico, entre otros, para que los emprendedores ofrezcan sus productos y servicios; así como la aplicación de técnicas estadísticas para generar políticas públicas, identificación de mercado y realizar predicciones que beneficien a los actores del TRC

\section{Agradecimientos}

Nuestro agradecimiento al Consejo Nacional de Ciencia, Tecnología e Innovación Tecnológica del Perú (CONCYTEC), Universidad Peruana Unión (filial Juliaca) y Grupo Innop Perú E.I.R.L. por el financiamiento de este trabajo de investigación.

\section{Referencias}

Buhalis, D. (2003). eTourism: information technology for strategic tourism management. Pearson. (Financial Times/Prentice Hall), London ISBN 0582357403.

Gómez, Rodolfo M. (2011). La era digital. Cómo la generación net está transformando al mundo. Culturales, 7(13), 177-183. Recuperado de de http://www.scielo.org.mx/scielo.php?script=sci_arttext\&pid=S1870-

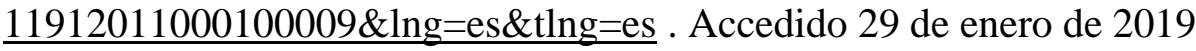

Maquera, G., Mariaca, J. y Mendoza, O. (2016). Informe indicador 5 de plataforma digital inteligente y big data para el turismo rural comunitario en la región Puno - Perú. UPeUCONCYTEC.

Kim, S., Kandampully, J. y Bilgihan, A. (2018). The influence of eWOM communications: An application of online social network framework. Computers in Human Behavior, 80, $243-$ 254.

Qiufen, Z. (2012). Research on Tourist Attractions Performance Promoting Method Based on the SWOT Analysis Method, IERI Procedia, 1, 254-260.

MINCETUR (2019). Turismo rural comunitario. Recuperado de https://www.mincetur.gob.pe/producto-turistico/turismo-rural-comunitario/

Osterwalder, A. y Pigneur, Y. (2009). Business Model Generation. Recuperado de http://www.businessmodelgeneration.com/.

Beck, K. y Andres, C. (2005). Extreme Programming Explained: Embrace Change. 2nd Edition (The XP Series). Pearson Education,

ISO/IEC 25010. ISO 25000 calidad del producto de software. Recuperado de https://www.iso25000.com/index.php/normas-iso-25000/iso-25010. 